\title{
IL RUOLO DEL CONCILIATORE NEL TRIBUNALE SPECIALE CIVILE SPECIALE PRESSO IL QUARTO TRIBUNALE CIVILE SPECIALE DI MACAPÁ-AP, AMAZÔNIA, BRASILE
}

\section{ARTICOLO ORIGINALE}

NETO, Dalk Dias Salomão ${ }^{1}$, SOUSA, Nicole Moreira Faria ${ }^{2}$, DENDASCK, Carla Viana $^{3}$, FECURY, Amanda Alves ${ }^{4}$, OLIVEIRA, Euzébio de ${ }^{5}$, DIAS, Claudio Alberto Gellis de Mattos $^{6}$

NETO, Dalk Dias Salomão. Et. II ruolo del conciliatore nel tribunale speciale civile speciale presso il quarto tribunale civile speciale di Macapá-AP, Amazônia, Brasile. Revista Científica Multidisciplinar Núcleo do Conhecimento. Anno 06, Ed. 05, Vol. 11, pp. 80-92. Maggio 2021. ISSN: 2448-0959, Link di accesso: https://www.nucleodoconhecimento.com.br/legge/tribunale-speciale, DOI: 10.32749/nucleodoconhecimento.com.br/legge/tribunale-speciale

\section{RIEPILOGO}

A causa di numerosi problemi sociali, la giustizia cominciò ad essere più richiesta dalla società. Era urgente mente necessario un nuovo paradigma, con la creazione di procedimenti giudiziari speciali, basati sulla conciliazione, come mezzo alternativo di risoluzione dei conflitti come soluzione parziale. I tribunali speciali si sono evoluti molto

\footnotetext{
${ }^{1}$ Avvocato, Bachelor of Law (CEAP - Center for Higher Education of Amapá), Specialista in diritto del lavoro e processo del lavoro dall'istituto educativo Damásio.

${ }^{2}$ Avvocato, Bachelor of Law (CEAP - Center for Higher Education of Amapá), specialista in diritto processuale civile dall'istituto Damásio Educacional.

${ }^{3}$ Teologo, PhD in Psicoanalisi Clinica. Si occupa da 15 anni di Metodologia Scientifica (Metodo di Ricerca) in Orientamento alla Produzione Scientifica per Master e Dottorandi. Specialista in Ricerche di Mercato e Ricerche Sanitarie Dottorando in Comunicazione e Semiotica (PUC SP).

${ }^{4}$ Biomedicale, PhD in Malattie Tropicali, Professore e ricercatore del Corso di Medicina del Campus Macapá, Università Federale di Amapá (UNIFAP), Prorettore di Ricerca e Studi Universitari (PROPESPG) dell'Università Federale di Amapá (UNIFAP).

${ }^{5}$ Biologo, PhD in Malattie Tropicali, Professore e ricercatore del Corso di Educazione Fisica, Università Federale di Pará (UFPA).

${ }^{6}$ Biologo, Dottore di Ricerca in Teoria e Comportamento, Professore e ricercatore del Corso di Laurea in Chimica dell'Istituto di Educazione Di Base, Tecnica e Tecnologica di Amapá (IFAP) e del Corso di Laurea in Formazione Professionale e Tecnologica (PROFEPT IFAP).

RC: 85945

Link di accesso: https://www.nucleodoconhecimento.com.br/legge/tribunale-speciale
} 
dalla sua creazione, attraverso la legge 9.099/95, che si è rivelata estremamente importante per la riorganizzazione della giustizia, di fronte alle grandi richieste di cause legali. L'obiettivo di questo lavoro era quello di analizzare il ruolo del conciliatore nel tribunale civile virtuale speciale nella 4avara del tribunale civile speciale di Macapá AP, Amazônia, Brasile. Si conclude che c'è stata un'evoluzione storica dei giudici speciali, dalla sua attuazione attraverso la legge 9.099/95 alla costruzione dei suoi principi come la rapidità procedurale, l'informalità e, il tutto indicando la realizzazione di un processo più rapido ed efficiente. PROJUD e TUCUJURIS informatizzando i tribunali speciali sembrano essere importanti per l'ammodernamento del processo, rendendoli più semplici, veloci e accessibili a tutti e seguendo così le tendenze tecnologiche mondiali. I tribunali speciali nel loro complesso dovrebbero cercare di investire sempre più in conciliatori e azioni finalizzate alla conciliazione, come le settimane di conciliazione statali e nazionali, perché in tal modo vi sarà una grande possibilità che i casi nel sistema giudiziario acquisisano maggiore fluidità.

Parole chiave: Tribunale civile speciale, conciliazione, conciliatore.

\section{INTRODUZIONE}

A causa di numerosi problemi sociali, la giustizia ha iniziato ad essere più richiesta dalla società, avendo problemi, come il gran numero di richieste procedurali, il ritardo nei processi di valutazione e la burocrazia (Sena e Richa, 2011; Guimarães, 2013).

Aggiungendo queste situazioni con la difficoltà di accesso delle persone con iposufficienza economica nella magistratura, c'era l'urgente necessità di un nuovo paradigma, avendo come soluzione parziale la creazione di tribunali speciali, basati sulla conciliazione, come mezzo alternativo di risoluzione dei conflitti (Sérpias, 2015).

I tribunali speciali in Brasile, precedentemente chiamati Small Claims Court, modellavano una struttura americana nota come Small Claims Courts. Creato nel 1934 a New York, con l'obiettivo di godere di azioni civili di piccolo valore. La caratteristica principale di questo modello di taglio è il pragmatismo proprio della cultura americana, cioè il conflitto visto sale a una prospettiva più rapida, semplice e facile. Non era 
necessaria la figura dell'avvocato, che è stato richiesto in base alla complessità della situazione (Porto, 2008).

Questo modello di tribunale è apparso in Brasile, prima a Rio grande do Sul, nel 1982, prima del funzionamento dei Consigli di conciliazione e arbitrato, creati con l'obiettivo di risolvere, extragiudiziale, conflitti di piccole cause, dimostrando un grande interesse per la costruzione di una giustizia (Silva, 2013).

Dopo aver preso risalto nello scenario nazionale, nel 1984, la magistratura ha avuto come pietra miliare la legge n. 7.244 , che prevede la creazione e il funzionamento di tribunali di piccole cause, consolidando e legittimando il successo dell'esperienza ottenuta con i Consigli di conciliazione e arbitrato, avendo come suo dovere, di fornire una giustizia paritaria (Silva, 2013).

Da quel momento in suso, diversi procedimenti del Tribunale speciale stanno iniziando a emergere in tutto il paese, regolati in ufficio statale dalla legge ordinaria, che coprono solo cause civili di piccolo valore economico, stabilite per la prima volta in un massimo di venti (20) salari minimi. Pertanto, ha anche permesso ai più privi finanziariamente dell'opportunità e della garanzia legale di cercare la magistratura al fine di ottenere una soluzione pacifica dei loro conflitti (Silva, 2013).

La Costituzione federale del 1988 ha dimostrato che la reale necessità di creare giudici speciali e la loro attuazione in tutto il paese è una misura obbligatoria, poiché in precedenza era facoltativa per gli Stati. II CF ha assicurato attraverso l'art. 98, punto I, l'autorizzazione ad attuare i Tribunali Civili Speciali per conciliare, perseguire, giudicare ed eseguire le cause civili di minore complessità, nonché speciali tribunali penali per riconciliare, giudicare ed eseguire reati di minore potenziale offensivo, attraverso procedure orali e molto sumaríssimo (che non ha ritardi, nessuna formalità) (Sérpias, 2015).

Dalla creazione della legge complementare n. 123/2006, con l'obiettivo di soddisfare i desideri dei micro e piccoli imprenditori circa la costituzione e la regolamentazione di ME e PPE, al fine di espandere le imprese e sviluppare la loro crescita nell'economia brasiliana. Così, il ME e il PPE hanno guadagnato spazio nello scenario nazionale e RC: 85945 Link di accesso: https://www.nucleodoconhecimento.com.br/legge/tribunale-speciale 
da questa legge generale è stata creata la disposizione giuridica sulla possibilità che queste specie di società, a livello statale, abbiano il diritto di entrare nel Tribunale civile speciale, comparendo nel polo attivo del ricorso (Salomão, 2001). Data la possibilità di accedere a un tribunale speciale, queste società possono inserire le loro azioni senza la presenza di un avvocato (con un massimale per il valore dell'azione fino a 20 salari minimi), riducendo le loro spese. È importante sottolineare che i casi nei tribunali speciali sono trattati più rapidamente che in tribunale comune, consentendo più rapidamente il recupero del capitale, che fino ad allora è stato perso a causa dell'inadempienza dei debitori (Tolentino, 2011).

Nel 2001, poco dopo la creazione della legge 9.099/95 (legge speciale), è stata creata l'estensione del tribunale civile per servire le microimprese ad Amapá, in considerazione del motivo che non potevano chiedere al Tribunale speciale centrale, che era l'unico in funzione in qualsiasi stato all'epoca, avendo come coordinatore il magistrato Sueli Pini, attualmente presidente della Corte di giustizia di Amapá (Serra, 2015).

Subito dopo la creazione dello statuto nazionale di ME e PPE, è stata creata la legge statale complementare n. 044/2007, in cui dimostra che i benefici della legge n. 123/06 sono stati accettati dal governo di Amapá, il Tribunale speciale delle microimprese e delle piccole imprese è stato finalmente creato nel marzo 2012 (Serra, 2015).

La corte delle microimprese, ad Amapá, ha un ruolo rilevante attraverso il lavoro sviluppato e il numero di riconciliazioni fruttuose. La maggior parte dei casi, già archiviati, sono dovuti ad approvazioni di accordi, sia giudizialmente che stragiudiziali. Raccontare principalmente con l'aiuto dei suoi collaboratori tirocinanti, che, nella maggior parte dei casi, sono i conciliatori nelle udienze. È importante notare che la $4^{\circ}$ corte del tribunale civile speciale di Macapá è quella che ha il maggior numero di tirocinanti di livello superiore, in considerazione delle loro partnership firmate come tjap, Fecomércio, FEMICRO e SEBRAE (Serra, 2015).

Pertanto, i tribunali speciali si sono evoluti molto dalla sua creazione, attraverso la legge 9.099/95, che si è rivelata estremamente importante per la riorganizzazione della 
giustizia, di fronte alle grandi richieste di cause legali. Oltre alla valorizzazione di ME e PPE, guadagnando spazio davanti ai tribunali speciali, per combattere in modo più dignitoso per i loro diritti, essendo la 4a corte del tribunale civile speciale di Macapá (ex corte di microimprese e piccola azienda di Macapá), pioniere in Brasile.

\section{OBIETTIVO}

Analizzare il ruolo del conciliatore nel tribunale civile virtuale speciale nella 4avara del tribunale civile speciale di Macapá AP, Amazônia, Brasile.

\section{METODO}

Ricerca quantitativa e qualitativa, utilizzando i dati della $4^{\circ}$ Corte del Tribunale Civile Speciale della città di Macapá, Amapá, Amazônia, Brasile, sulla conciliazione nei procedimenti giudiziari.

\section{RISULTATI E DISCUSSIONE}

I tribunali civili speciali sono organi che coevengono la giustizia ordinaria e sono stati creati con lo scopo di conciliazione, processo, processo ed esecuzione, nelle cause della loro giurisdizione, disciplinati dalla legge federale n. 9,099 del 26 settembre 1995. Le basi di questi procedimenti sono guidate dai criteri di oralità, semplicità, informalità, economia procedurale e rapidità, mirando, ove possibile alla conciliazione, al risarcimento del danno subito dalla vittima e all'applicazione di una pena non detentiva (Neto e Júnior, 2011).

La vera intenzione dei tribunali civili speciali è la risoluzione dei problemi di accesso alla giustizia, fondamentalmente legati ai costi e al ritardo dei casi, e ci sono anche numerosi ostacoli che i cittadini, che chiedono giustizia, incontrano (Guimarães, 2013).

I giudici civili speciali sono sempre più presenti nella vita di tutta la popolazione, poiché la loro esistenza è giustificata. È presentato dal suo modo moderno di agire, dalla qualità del servizio e dagli interessi dei suoi leader che li rende sempre più agili e accessibili (Bacellar, 2003). 
II Tribunale civile speciale è competente per la conciliazione, il procedimento e la sentenza di cause civili di minore complessità, sono esempi: cause il cui valore non supera il quaranta volte il salario minimo; quelli elencati all'art. l'azione di sfratto per uso proprio; azioni di possesso su immobili di valore non superiore a quaranta volte il salario minimo; titoli esecutivi extragiudiziali (cambiali, assegni, contratti), per un importo massimo di quaranta volte il salario minimo; risarcimento dei danni (ad esempio danni derivanti da incidenti stradali) (Barouche, 2010).

\section{PRINCIPI DEI GIUDICI CIVILI SPECIALI}

La creazione di giudici speciali è un'importante pietra miliare per il mondo giuridico in tutti i suoi ambiti, rendendo necessario evidenziare i principi che guidano questo ordine, sia nella ricerca del suo significato, sia nella portata e applicabilità della norma giuridica (Porto, 2008).

Secondo Sérpias (2015) "lo scopo della creazione di un tribunale civile speciale è quello di risolvere rapidamente ed economicamente le piccole questioni del cittadino nella giornata quotidiana". Pertanto, la legge è stata creata sulla base di principi compatibili con l'agevolazione dell'accesso alla giustizia di coloro che la cercano.

\section{PRINCIPIO DI ORALITÀ}

Grazie allo stampo moderno dei giudici speciali, il principio di oralità si distingue per la sua facile applicabilità. Questo principio è incluso tra i generali dei procedimenti civili, con l'obiettivo di prevalere gli atti procedurali attraverso la comunicazione orale, sebbene tali atti possano essere ridotti alla scrittura (Silva, 2012).

Nei corti dei corti dei casi questo principio è molto più presente e solo l'essenziale è ridotto a termine. II resto può essere inciso su nastro magnetico o equivalente, ai sensi dell'articolo 13, §3, della legge n. 9.099/95 (Brasile, 1995). Ciò dimostra che l'oralità non ha sostituito la scrittura, che vanno entrambe di pari passo, poiché è essenziale rendere il processo e la conversione dei loro atti procedurali in termini. 
L'applicazione di questo principio mira anche a far eseguire atti procedurali in un'unica fase $o$ in momenti approssimativi. Pertanto, questo principio si riferisce direttamente alla rapidità procedurale, poiché non dovrebbe esserci un divario di tempo molto ampio tra le pratiche procedurali (Silva, 2012). Pertanto, questo principio mira alla semplicità procedurale, come mezzo per facilitare il progresso del processo, culminando nella sua rapidità.

\section{PRINCIPIO DI INFORMALITÀ E SEMPLICITÀ}

II nome stesso di questi principi dimostra già la sua intenzione, mirano a facilitare il processo e l'accesso alla magistratura, non mantenendo la forma stessa, ma piuttosto a una maggiore flessibilità degli atti processuali, convalidandoli ogni volta che raggiungono il loro scopo, secondo (art. 13 della legge 9.099/95): "Art. 13 - Gli atti processuali saranno validi ogniqualvolta soddisfino le finalità per le quali sono eseguiti, soddisfacendo i criteri di cui all'art.

II principio di semplicità non è altro che uno svolgimento del principio di informalità, che a sua volta è il tentativo di rendere le procedure giudiziarie più informali, senza quell'esacerbazione di formalismi e burocrazie. Secondo Bobbio, lo zelo per la burocrazia processuale piuttosto che garantire la piena efficacia dell'approvvigionamento giudiziario ha finito per confluirsi nel ritardo del procedimento (Porto, 2008).

Un esempio pratico di questi principi è il modo in cui la citazione postale è fatta di persone giuridiche di diritto privato, essendo valida con la semplice consegna della corrispondenza a qualsiasi dipendente responsabile dell'accoglienza (art. 18, punto II, della legge n. 9.099/95), a differenza del codice di procedura civile che prevede la consegna a determinate persone, ad esempio quelle che hanno poteri di gestione o di amministrazione; (art. 18, § 2 della Legge 9.099/95) (Brasile, 1995): "Art. 18 - La quotazione deve esser[...]e fatta: II - nel caso di una persona giuridica o di una singola impresa, mediante consegna al responsabile dell'accoglienza, che deve essere necessariamente identificata". 
Pertanto, questi due principi con l'obiettivo principale di portare la popolazione più semplice al forum, poiché la parte più indigena della popolazione e dei laici negli affari legali si sente timida di fronte ad avvocati, giudici, formalismi e persone con vocabolari più inverosimile.

\section{PRINCIPIO DELL'ECONOMIA PROCEDURALE}

Un altro principio è quello dell'economia procedurale, che a sua volta è una guida non solo del tribunale speciale, ma della teoria generale del processo nel suo complesso, poiché attraverso di esso ha come obiettivo principale quello di raggiungere il maggior numero di risultati con la spesa economica più bassa possibile (Sérpias, 2015).

Pertanto, questo principio mira a che il maggior numero di atti processuali venga eseguito nel più breve tempo possibile e nel modo meno costoso possibile.

\section{PRINCIPIO DELLA RAPIDITÀ PROCEDURALE}

Per quanto riguarda il principio di rapidità, ciò avviene per garantire l'efficacia della giustizia, con l'obiettivo di rendere gli effetti di una sentenza, ad esempio, in modo pratico e rapido e non in modo lento come accade in molti casi, soprattutto al di fuori dei tribunali speciali (Sérpias, 2015)

Secondo Sérpias (2015) "Il ritardo nella disposizione giudiziaria è diventato una regola quando dovrebbe essere un'eccezione. II ritardo della fine del processo è stato istituzionalizzato. Pertanto, gli altri principi non sono altro che una conversione al principio della velocità, allo scopo di accelerare il processo.

Pertanto, senza dubbio, la più alta aspettativa generata dalla legge 9.099/95 è la promessa di velocità senza violare il principio di sicurezza degli scarichi legali. Con l'intenzione di rendere il processo più agile e rapido, e solo in questo modo raggiungere il suo scopo principale, una risposta efficiente dell'offerta giudiziaria alla società. 


\section{INFORMATIZZAZIONE DI GIUDICI CIVILI SPECIALI- PROJURD}

II mondo ha vissuto un grande progresso tecnologico negli ultimi anni, alleato con la mancanza di spazio fisico per custodire numerosi processi, soprattutto dopo la costituzione del 1988 e l'internazionalizzazione dell'economia con la creazione del reale, fattori che hanno sfruttato il numero di cause legali nella magistratura. In questo modo, la magistratura brasiliana ha creato la società PROJUDI (processo giudiziario digitale) (Silva, 2012).

Lo scopo principale della creazione di PROJUDI mira ad accelerare la giustizia, ridurre i costi, aumentare la capacità di trattamento dell'azione, facilitare il lavoro degli avvocati e migliorare la qualità del servizio alle parti. Così, può terminare quegli scaffali pieni di carte, poiché nei decenni precedenti i processi sono stati fatti quasi a mano, consentendo una riduzione dei tempi di attesa per l'apprezzamento delle richieste, cercando sempre di risolvere i conflitti più rapidamente senza causare danni alle parti.

La vera rivoluzione è iniziata con la Legge 11.419/2006, con l'intento di disciplinare il processo elettronico, con profondi cambiamenti nel Codice di Procedura Civile, e con l'obiettivo di snellire, potenziare, accorciare il percorso creato dalla burocrazia e la distanza sempre comune nei processi. Possiamo vedere che due campi si sono evoluti in modo significativo con l'informatizzazione, l'agilità e la velocità procedurale, perché i processi vengono tutti trasmessi via Internet senza dover lasciare l'ufficio (Carvalho, 2006).

Nella questione dell'informatizzazione, lo stato di Amapá è andato oltre, nel 2005, ha creato il sistema TUCUJURIS, uno dispositivo tecnologico che cerca di gestire e sviluppare elettronicamente le attività della magistratura. Informatizzazione delle procedure giudiziarie e svolgimento nelle Segreterie Giudiziarie, nonché archiviazione dei contenuti dei vari atti processuali, puntando così a una maggiore agilità dei servizi, sostituendo l'allora attuale sistema (Tjap, 2016).

II sistema TUCUJURIS è migliorato nel corso degli anni e le sue procedure sono migliorate sempre di più, con aggiunte di nuove funzionalità che lo rendono un software 
più integrato nell'ambito della giustizia, diversificando la sua operatività, con l'obiettivo di dare maggiore efficienza all'esecuzione di atti giurisdizionali da parte dei Server, Magistrati, Avvocati Difensori e Procuratori, nonché una risposta più rapida ad altri membri della società che utilizzano questo servizio (Tjap , 2016).

Pertanto, l'informatizzazione è indispensabile per il mantenimento di una giustizia rapida ed efficace, perché rende moderna la magistratura, seguendo le tendenze tecnologiche di oggi.

\section{CONCILIAZIONE NEI GIUDICI SPECIALI CIVEIS}

L'istituto di conciliazione è noto alla stragrande maggioranza dei brasiliani. Tuttavia, la sua applicazione nel campo del diritto, legata a questioni applicate alla giustizia conciliante, sembra ancora nuova per molti (Guimarães, 2013).

Sebbene la conciliazione sia presente nell'ordinamento giuridico brasiliano sin dai tempi dell'impero, è stato negli ultimi decenni che questo istituto ha guadagnato notorietà. Dall'emancipazione politica del Brasile nel 1822, la costituzione dell'impero ha apertamente incoraggiato la risoluzione dei conflitti con mezzi extragiudiziari, e la conciliazione è una condizione preventiva e indispensabile per il trattamento di qualsiasi causa, con la figura del giudice di pace come principale mezzo per risolvere il contenzioso (Silva, 2013).

Nel 1982 è stata pubblicata la legge dei tribunali speciali per le piccole cause, allo scopo di sburocratizzare il potere giudiziario a livello nazionale. Creato nel 1984, questo tribunale definitivamente, con la legge 7.244/84 (Sérpias, 2015).

Con l'emanazione di questa legge, la conciliazione ha ancora una volta risalto nella risoluzione dei conflitti, incluso l'inserimento della figura del conciliatore, che sarà studiata in seguito (Guimarães, 2013).

La conciliazione è un metodo utilizzato in conflitti meno complessi, in cui il terzo facilitatore può adottare una posizione più attiva ma neutrale rispetto al conflitto, non perdendo imparzialità. Si tratta di un breve processo consensuale che cerca una RC: 85945

Link di accesso: https://www.nucleodoconhecimento.com.br/legge/tribunale-speciale 
risoluzione efficace dei conflitti, entro i possibili limiti, del rapporto sociale tra le parti coinvolte (Sérpias, 2015).

In sintesi, "la conciliazione è un istituto del processo, perché è uno dei suoi momenti che produce effetti procedurali, privilegia il principio curioso e il ruolo del giudice interveninte nella formazione della volontà delle parti" (Nassif, 2005).

La conciliazione è un processo auto-compiacente in cui un terzo imparziale (il conciliatore) assiste le parti in conflitto al fine di trovare una soluzione soddisfacente per tutte le parti (Silva, 2013).

La conciliazione in sede di $4^{\circ}$ tribunale del tribunale civile è piuttosto sviluppata e praticata, essendo un tribunale di questo tipo noto a livello nazionale per il lavoro sviluppato e il numero di riconciliazioni positive. La maggior parte dei casi già presentati sono frutto di approvazioni di accordi, sia giudizialmente che stragiudiziali (Serra, 2015).

Possiamo quindi concludere che l'Istituto di conciliazione è senza dubbio il mezzo alternativo di risoluzione dei conflitti più utilizzato e noto in Brasile, che consente alla giustizia di risolvere rapidamente le sue richieste, oltre a rendere reale la possibilità di una soluzione pacifica dei paesi.

\section{FASI DI CONCILIAZIONE}

La conciliazione, per quanto informale, ha alcuni passi che devono essere osservati dal conciliatore nella costruzione di un accordo, passi che non sono regole. Tuttavia, il rispetto di questi passaggi aiuta nella velocità e nell'efficacia del processo (Sérpias, 2015).

Pertanto, la conciliazione ha orientamenti da rispettare e non dovrebbe avvenire per semplice volontà delle parti o del conciliatore. Di seguito citeremo le principali fasi della conciliazione. 


\section{IL CONCILIATORE E LE SUE CARATTERISTICHE NEL TRIBUNALE CIVILE VIRTUALE}

La domanda interessante in questo momento è la figura del conciliatore nelle procedure della legge 9.099/95. I conciliatori saranno preferibilmente assunti da studenti di diritto. Quindi, se la legge ha detto preferibilmente, non lo rende un obbligo. Così, gli studiosi di legge possono anche figurare come conciliatori. Ai sensi della legge 9.099/95: "Art. 7. I conciliatori e i giudici onorari sono ausiliari di giustizia, assunti, il primo, preferibilmente, tra gli studenti di giurisprudenza, e il secondo, tra gli avvocati con più di cinque anni di esperienza" (Brasile, 1995).

In pratica, la stragrande maggioranza dei conciliatori del Tribunale civile speciale sono studenti di legge, estratti dalle università di solito dopo essere entrati nel quinto semestre. Essere una partnership, dove si scambia lavoro per esperienza, non essere diversi nella $4^{\circ}$ corte del tribunale civile speciale (Serra, 2015).

Il conciliatore è uno dei temi della conciliazione e deve agire in modo neutrale e imparziale, creando un momento e una situazione le cui parti possono sentirsi a proprio agio nel negoziare. Deve usare la sua psicologia per rendere fruttuosa la conciliazione, attraverso la volontà delle parti opposte, cioè il conciliatore non è altro che un facilitatore (Porto, 2008).

Anche portando tutto l'onere emotivo, durante l'audizione di conciliazione il conciliatore deve essere il più imparziale possibile e neutrale, deve garantire la fluidità e la legittimità del processo (Silva, 2013). L'imparzialità del conciliatore è fondamentale per la conciliazione, il che significa che non onorerà nessuna delle parti, tanto meno emanerà un giudizio di valore legato al conflitto. Dovrebbe quindi trasmettere fiducia alle parti. Solo in questo modo è possibile costruire un accordo equo per tutti (Amaral, 2011).

In relazione alla $4^{\circ}$ corte del tribunale civile speciale, essendo molto famosa per il numero di riconciliazioni fruttuose, ottenute in udienze quotidiane o eventi tenuti RC: 85945 Link di accesso: https://www.nucleodoconhecimento.com.br/legge/tribunale-speciale 
sporadicamente come: settimana di conciliazione statale o nazionale e "Sabato è anche giorno conciliante", si distingue per l'efficienza dei suoi conciliatori, e la maggior parte sono tirocinanti di livello superiore di diritto, date le loro partnership firmate tra il tribunale stesso e il TJAP , Fecomércio, FEMICRO e SEBRAE (Serra, 2015).

Pertanto, la figura del conciliatore di fronte alla conciliazione è fondamentale, perché è lui che organizza e conduce il lavoro. Deve agire in conformità con la legge e gli orientamenti da rispettare.

\section{L'EFFICIENZA DELLA CONCILIAZIONE}

Non solo la mancanza di efficacia e la rapidità dei principali problemi di giustizia, ma anche altre situazioni contribuiscono a ritardare e fermare il sistema giudiziario. Pertanto, attraverso la Costituzione federale del 19888 e regolata dalla legge 9.099/95, i tribunali civili speciali sono emersi come un modo per espandere l'accesso alla giustizia ed è stato caratterizzato come una "valvola di fuga" alla giustizia comune, cambiando le procedure tradizionali che erano inefficaci, specialmente per la risoluzione di piccoli conflitti (Guimarães, 2013).

La creazione di tribunali speciali e il ricorso alla conciliazione hanno un'efficacia ben al di là di quanto ci si aspetta, perché oltre a erogare la magistratura ha portato alla portata della giustizia i cittadini che fino ad allora non hanno usato questo mezzo per risolvere i loro conflitti, diffondendo così il diritto e la cultura della pace (Guimarães, 2013).

Così, nel corso degli anni la conciliazione si è dimostrata efficace, poiché non ha preso in considerazione la magistratura, attraverso la riduzione dei costi, i termini e la risoluzione delle cause di minore complessità, creando nuovi parametri per la risoluzione dei conflitti nella giustizia nel suo complesso, tenendo conto della rapidità (Guimarães, 2013).

Pertanto, la maggiore velocità dei giudici speciali si basa sull'idea di velocità ed efficacia e ci sono due modi per suddividere l'efficienza, qualitativa e quantitativa. II quantitativo non riguarda la qualità procedurale, tenendo conto solo della rapidità delle RC: 85945 Link di accesso: https://www.nucleodoconhecimento.com.br/legge/tribunale-speciale 
procedure e della riduzione dei costi, mentre qualitativo, al contrario, si occupa del contenuto delle decisioni, tenendo conto della loro qualità in materia di rispetto e adozione del processo democratico, di tutte le norme ad esso inerenti (Neto, 2015).

In questo senso, possiamo dire che, sebbene tribunali speciali e conciliazione siano efficaci, dare rapidità ai loro procedimenti non significa che i problemi del sistema giudiziario siano stati risolti, tanto meno possiamo dire che la giustizia nel suo complesso è rapida (Barouche, 2010).

Per quanto riguarda Macapá, i giudici speciali sono molto efficaci, dato l'incentivo alla conciliazione (Serra, 2015).

Ad esempio, la 4a corte del tribunale speciale di Macapá, ex tribunale delle microimprese e delle piccole imprese, ha un lavoro molto concreto ed efficace utilizzando la conciliazione, essendo riconosciuto a livello nazionale per il suo lavoro che si traduce ogni anno in fruttuose riconciliazioni (Serra, 2015).

Nel corso dell'anno vengono condotte numerose azioni di conciliazione, come ad esempio settimane di conciliazione statali e nazionali. In esse, la $4^{\circ}$ corte del tribunale civile speciale di Macapá è sempre quella che fa le riconciliazioni più fruttuose (Serra, 2015).

La parte fisica del tribunale limita indubbiamente notevolmente il numero di conciliazioni, ma anche così si tengono da 12 a 25 udienze di conciliazione al giorno, un numero che è un numero più alto rispetto agli altri tribunali speciali della corte centrale di Macapá (Serra, 2015). 
Figura 1- Indica il numero di cause relative alla conciliazione, all'istruzione e alla sentenza

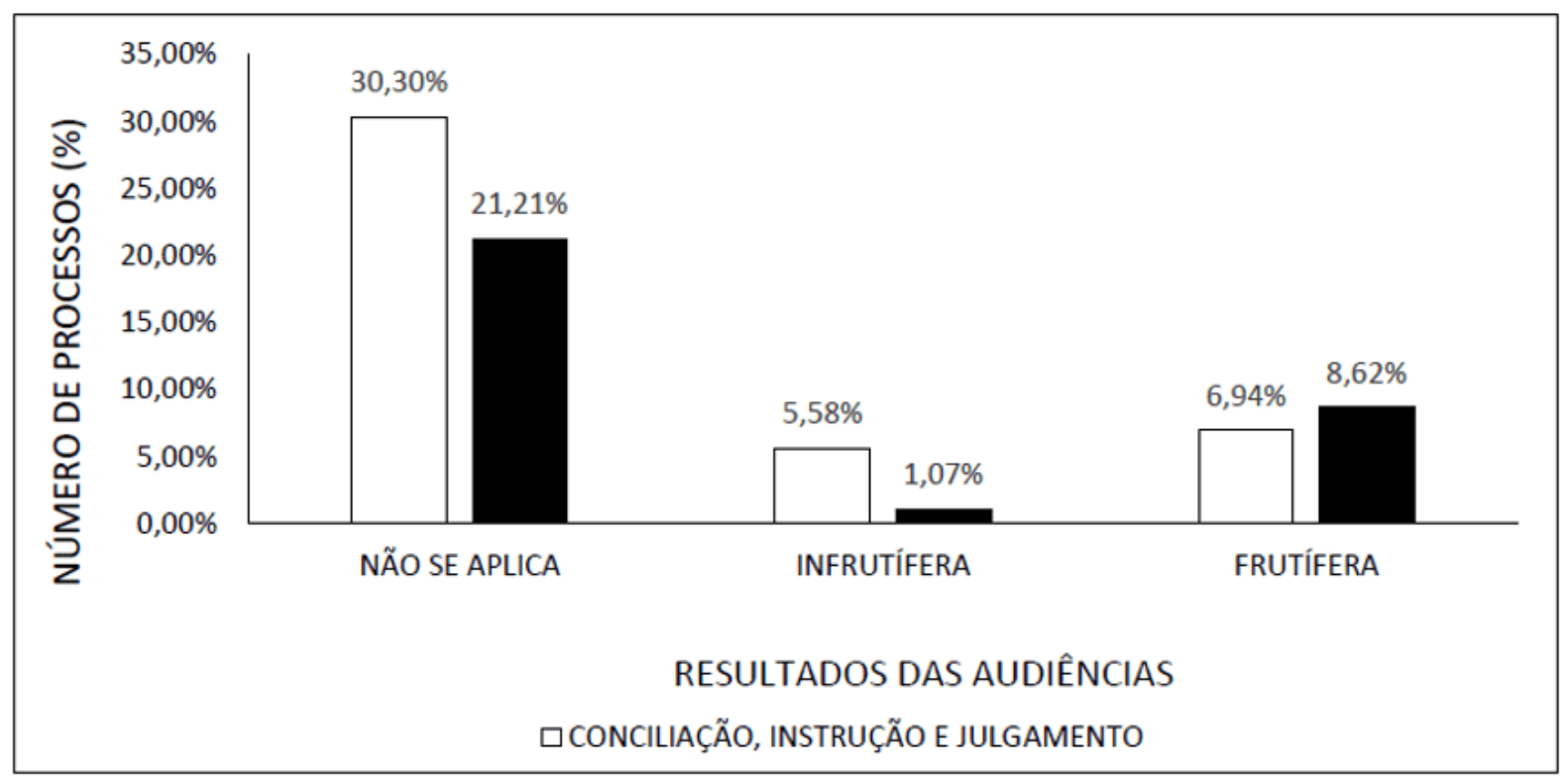

(Serra, 2015).

La figura 1 mostra l'efficienza delle udienze di conciliazione tenute presso il $4^{\circ}$ tribunale del tribunale civile speciale di Macapá. Durante le audizioni inaugurali di conciliazione la percentuale di accordi fruttuosi è più elevata; e l'accordo fallito è minore (data la presenza del conciliatore), in relazione alla fase di istruzione e giudizio, davanti al giudice.

Pertanto, possiamo concludere che l'obiettivo della creazione di tribunali speciali è stato raggiunto dinanzi al $4^{\circ}$ tribunale del tribunale civile speciale di Macapá, perché, attraverso la conciliazione, il numero di richieste risolte attraverso accordi è significativo rispetto alle altre fasi procedurali. Dimostrando così l'efficacia del conciliatore come motore delle audizioni di conciliazione.

\section{CONCLUSIONI}


In considerazione dell'aumento del numero di casi, il sistema giudiziario sembra essere diventato congestionato e inefficiente, ed è necessario cercare nuovi mezzi per la risoluzione dei conflitti. La risposta del potere giudiziario è stata la creazione di tribunali speciali mediante conciliazione.

C'è stata un'evoluzione storica dei giudici speciali, dalla sua attuazione fino alla legge 9.099/95 alla costruzione dei suoi principi come la rapidità procedurale, l'informalità e, il tutto indicando la realizzazione di un processo più rapido ed efficiente.

PROJUD e TUCUJURIS informatizzando tribunali speciali sembrano essere importanti per modernizzare il processo, rendendoli più semplici, veloci e accessibili a tutti e seguendo così le tendenze tecnologiche globali

L'istituto di conciliazione si rivela il principale mezzo alternativo di risoluzione dei conflitti, una forma di autoco composizione, attraverso la quale le parti creano rapidamente la soluzione ai problemi, sprocendo rapidamente il sistema giudiziario.

La conciliazione attraverso la rapidità, i bassi costi e l'efficienza sembra rendere il sistema giudiziario più attraente per parte della società a basso reddito e per i laici in materia, che credono nella possibilità di risolvere i loro problemi alla base della giustizia, generando così effetti positivi sulla pacificazione sociale.

L'evoluzione dei procedimenti speciali, basata sulla conciliazione, non sarebbe efficace senza la presenza del conciliatore. È lui che dirige le parti alla stesura di un accordo, conducendo in modo imparziale, guidando la procedura su numerosi principi e orientamenti. Pertanto, si dovrebbe essere maggiormente interessati alla formazione di questi agenti, poiché sono loro che sono in prima linea nella maggior parte delle richieste della magistratura, che affrontano direttamente e quotidianamente la popolazione e i suoi problemi, in situazioni stressanti e, a volte, difficili da conciliare.

I tribunali speciali nel loro complesso dovrebbero cercare di investire sempre più in conciliatori e azioni finalizzate alla conciliazione, come le settimane di conciliazione statali e nazionali, perché in tal modo vi sarà una grande possibilità che i casi nel sistema giudiziario acquisisano maggiore fluidità. 


\section{RIFERIMENTI}

AMARAL, R. L. S. D. A Concilição no Processo Judicial, . 2011. 64 (Graduação). Faculdade de Ciências Jurídicas da Universidade Tuiuti do Paraná, Curitiba PR.

BACELLAR, R. P. Juizados Especiais - a Nova Mediação Paraprocessual. São Paulo: Revista dos Tribunais, 2003. 286.

BAROUCHE, T. D. O. Os Juizados Especiais Cíveis e a Problemática da Celeridade Processual. Revista de Direito dos Monitores da UFF, n. 7, p. 1-24, 2010.

BRASIL. LEI № 9.099, DE 26 DE SETEMBRO DE 1995. JURÍDICOS, S. P. A. Brasília DF: Presidência da República - Casa Civil 1995.

CARVALHO, J. P. C. D. A Era Virtual do Processo Judicial: A Experiência dos Juizados Especiais Virtuais e o Projeto de Lei de Informatização do Processo. Revista Direito e Liberdade, v. 3, n. 2, p. 453 - 484, 2006.

GUIMARÃES, A. A Crise do Judiciário e a Eficácia da Conciliação nos Juizados Especiais Cíveis Estaduias de São Luis - MA. Revista Thesis Juris, v. 2, n. 1, p. 142161,2013

NASSIF, E. Conciliação judicial e indisponibilidade de direitos: paradoxos da "justiça menor" no processo civil e trabalhista. São Paulo SP: Editora LTR, 2005.

NETO, A. G. A Audiência de Conciliação no Novo Código de Processo Civil. Revista Eletrônica de Direito Processual - REDP. 16, n. 16, p. 29-57, 2015.

NETO, F. D. C. T.; JÚNIOR, J. D. F. Juizados Especiais Estaduais Cíveis e Criminais. Comentários à Lei no 9.099/95. $7^{a}$ ed. São Paulo: Revista dos Tribunais, 2011. 960p. 
PORTO, A. P. G. Juizados Especiais Cíveis - O Papel do Conciliador 2008. 59p. (Especialização). Universidade Estadual Vale do Acaraú, Fortaleza CE.

SALOMÃO, L. F. As Microempresas nos Juizados Especiais. Revista da EMERJ, v. 4, n. 4, p. 62-73, 2001.

SENA, A. G.; RICHA, M. D. A. A conciliação judicial trabalhista em uma política pública de tratamento adequado e efetivo de conflito de interesses. In: PELUSO, A. C. e RICHA, M. D. A. (Ed.). Conciliação e mediação: estruturação da política judiciária nacional. Rio de janeiro RJ: Forense, 2011. p.115-139.

SÉRPIAS, L. Juizado Especial, Cível, Criminal, Federal e da Fazenda Pública. São Paulo: TradeBook, 2015. 1615p.

SERRA, A. B. C. S. Juizado Especial da Microempresa e Empresa de Pequeno Porte: Um Estudo de Caso na Cidade de Macapá. 2015. 18p. (Graduação). Estácio de Sá, Macapá AP.

SILVA, É. B. Conciliação Judicial. Brasília DF: Gazeta Juridica, 2013. 432p.

SILVA, P. G. A Conciliação como Instrumento de Solução de Conflitos. 2012. (Graduação). Centro Universitário Eurípides de Marília - UNIVEM, Marília SP.

TJAP. Sistema TUCUJURIS - Processo Judicial Eletrônico. Macapá AP, 2016. Disponível em: < http://www.tjap.jus.br/portal/sobre.html >. Acesso em: 22/02/2016.

TOLENTINO, A. V. A competência do Juizado Especial Cível. 2011. Disponível em: $<\quad$ http://www.direitonet.com.br/artigos/exibir/6387/A-competencia-do-JuizadoEspecial-Civel >. Acesso em: 30/05/2016.

Inviato: Maggio 2021.

Approvato: Maggio 2021. 